\title{
Las tecnologías de la información y comunicación como factor de innovación y competitividad empresarial
}

\author{
Information and communication technologies as a factor of innovation and
}

\author{
M. C. Bernal-Jiménez , D. L. Rodríguez-Ibarra iD
}

Resumen - En la gestión empresarial las tecnologías de la información y comunicación son una excelente herramienta que genera un valor agregado a las actividades operacionales. De igual forma, la innovación es un conjunto de procesos que buscan la optimización o creación de nuevos productos o servicios. En el siguiente artículo de revisión se realizó un estudio documental bajo un enfoque cualitativo-descriptivo sobre el efecto que genera las tecnologías de la información y comunicación y la innovación en la competitividad empresarial. Se mencionan algunos casos de éxito que llevó a diferentes empresas a posicionarse a nivel nacional e internacional y por medio de una triangulación se identificaron los factores que deben tener en cuenta las empresas que incorporen estrategias de innovación utilizando las tecnologías de la información y comunicación para alcanzar éxito en la competitividad empresarial.

Palabras clave - Competitividad Empresarial, Innovación, Tecnologías de la información y comunicación.

\begin{abstract}
In business management, information and communication technologies are an excellent tool that generates added value for operational activities. Similarly, innovation is a set of processes that seek the optimization or creation of new products or services. In the following review article, a documentary study was carried out under a qualitative-descriptive approach on the effect generated by information and communication technologies and innovation in business competitiveness. Some successful cases are mentioned that led different companies to position themselves at a national and international level and by means of a triangulation, the factors that should be taken into account by companies that incorporate innovation strategies using information and communication technologies were identified to reach success in business competitiveness.
\end{abstract}

Index Terms - Business Competitiveness, Innovation, Information Technology and Communication

Este manuscrito fue enviado el 08 de diciembre de 2018 y aceptado el 26 de marzo de 2019. Es un artículo de investigación científica del proyecto en desarrollo denominado "Estrategias de innovación tecnológica en empresas del sector turismo del departamento Norte de Santander para el mejoramiento de la competitividad. Caso de estudio municipio de Durania" el cual hace parte de un proyecto de investigación que forma parte la convocatoria de jóvenes investigadores de Colciencias del año 2018.

\section{INTRODUCCIÓN}

$\mathrm{D}$ EBIDO a la globalización la exigencia empresarial ha demandado que las empresas incrementen sus niveles de competitividad, siendo esta la capacidad empresarial de generar productos o servicios con calidad y eficiencia en el mercado, que les permita posicionarse a nivel nacional e internacional. ¿Pero cómo lograr la competitividad empresarial? Hoy en día se observa como las empresas se preocupan por implementar diferentes estrategias con el fin de mejorar su productividad y optimizar sus procesos.

La implementación de las tecnologías de la información y comunicación (TIC) se ha convertido en una excelente herramienta que permite a las empresas generar un valor agregado a las actividades operacionales, buscando ofrecer ventajas empresariales para lograr consolidarse en un mercado global. De igual forma se puede evidenciar, como las empresas han optado por la innovación siendo este un conjunto de herramientas que permiten aplicar de forma correcta la gestión del conocimiento.

En consecuencia, el presente artículo realiza un análisis de la competitividad empresarial y los factores que influyen en el éxito de la misma tomando en cuenta las tecnologías de información y comunicación. Para ello, se realizó un proceso investigativo que se desarrolló por medio del enfoque cualitativo-descriptivo realizando una revisión a través del método de triangulación de datos con el fin de efectuar un análisis de los casos de éxitos empresariales e identificar los factores que influyen en una correcta implementación de la innovación y las tecnologías de la información y comunicación.

M. C. Bernal-Jiménez, Programa de Ingeniería de Sistemas, Universidad Simón Bolívar, Cúcuta, Colombia. m.bernal@unisimonbolivar.edu.co.

D. L. Rodríguez-Ibarra, Programa de Ingeniería de Sistemas, Universidad Simón Bolívar, Cúcuta, Colombia.d_rodriguez9@unisimon.edu.co 


\section{CONCEPTOS FUndAMENTALES}

\section{A. La competitividad empresarial}

La competitividad es la capacidad empresarial de brindar productos y servicios con eficiencia y calidad en el mercado, actuando como un conjunto de estrategias que permiten alcanzar las metas trazadas por las empresas. La competitividad requiere no solo la atención en la productividad, también se deben fortalecer los procesos externos e internos de la empresa, logrando como resultado un posicionamiento en el mercado cada vez más amplio [1]. Para este fortalecimiento la competitividad propone la combinación de un conjunto de aspectos que se interrelacionan y permiten alcanzar un alto nivel de desempeño empresarial, donde se deben incluir:

- Los procesos que son un conjunto continúo de trabajo que interactúan para alcanzar los objetivos comunes. ¿Pero en el ejercicio empresarial que es un proceso? Cuando una empresa ofrece bienes o servicios, debe plantear un proceso que tenga como destino final el consumidor. Cuando se piensa en la atención de un concesionario de automóviles, el proceso empresarial es el paso a paso del intercambio que tendrá el cliente y el vendedor, desde el momento que llega, hasta el momento posterior de la venta.

- Los recursos que son todos aquellos elementos que contribuyen al funcionamiento de la empresa, estos pueden ser recursos humanos, financieros, materiales y tecnológicos.

- Y la productividad que es cuando una empresa logra relacionar el resultado de una serie de procesos y los recursos que han sido utilizados para obtener dicho producto, lo que lleva a conseguir los objetivos trazados por la misma.

De esta manera, la competitividad es un factor fundamental en las empresas ya que permiten mejorar su posición frente al mercado global, logrando que tomen buenas decisiones ante las amenazas y riesgos que puedan presentar. Además, permite que

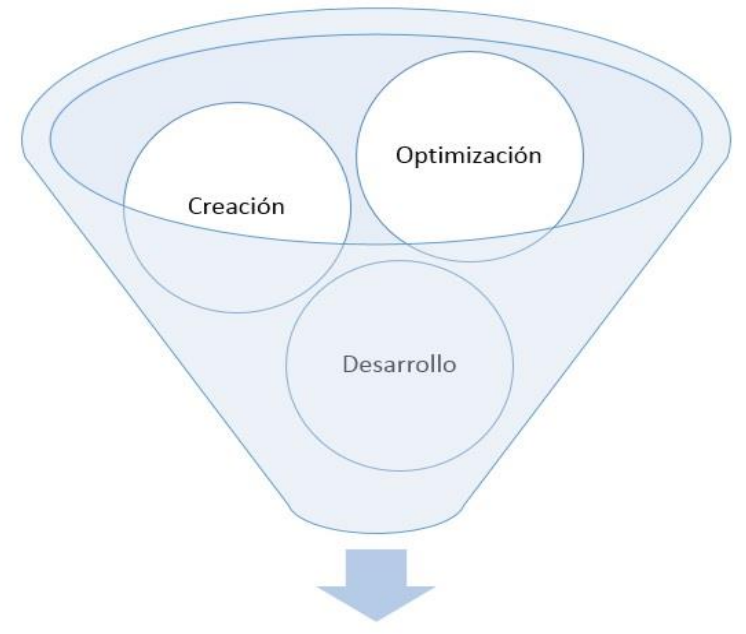

\section{Innovación}

Fig. 1. Modelo Conceptual de Innovación aumenten sus ingresos económicos gracias al rendimiento ofrecido a los inversores. Ibarra, Gonzáles y Demuner, afirman que es importante que las empresas empiecen a incluir la competitividad en su cultura empresarial, para que finalmente pueden alcanzar a ser competitivas a escala nacional e internacional y puedan aprovechar el conocimiento y los recursos para obtener una mayor productividad en el mercado [2].

Uno de los aspectos importantes para mejorar la competitividad empresarial es la innovación. Actualmente las empresas incorporan la innovación como la creación de nuevos procesos que buscan optimizar los productos y servicios de calidad. La innovación es la adopción de estrategias en los recursos humanos, técnicos y económicos con el fin de optimizar los resultados de la productividad de las empresas [3]. El mercado actual exige a las empresas rediseñar sus estrategias, e imponen la implementación de procesos innovadores que permitan la optimización de sus procesos y maximizar así su productividad. En la figura 1 se muestra la relación entre la optimización, la creación y el desarrollo con el fin de crear innovación.

La innovación no es un producto que se dispara bajo un momento de inspiración, es un conjunto de procesos que generan un producto. De esta manera, la creación es uno de los procesos que permiten que la empresa genere valor a través de la utilidad o riqueza, de igual forma la optimización se preocupa por la eficiencia de los recursos y finalmente el desarrollo hace referencia al progreso que la empresa adquiere mediante la evolución a lo largo del tiempo. Los procesos anteriormente mencionados son un factor esencial en la elaboración de la innovación, siendo esta un producto más complejo.

\section{B. ¿Por qué y para que innovar?}

La innovación permite generar y crear valor, es importante que constantemente las empresas piensen en cómo, para qué y de qué forma pueden optimizar sus procesos. La innovación empresarial permite que se realicen diferentes cambios, los cuales pueden ser organizacionales, productivos o tecnológicos contribuyendo a que se logre:

1. Transformación: serie de cambios que son necesarios, con el fin de mejorar la productividad de las empresas. La transformación involucra toda la organización que va desde los recursos tecnológicos hasta los recursos humanos para así lograr un crecimiento.

2. Crecimiento: Cuando la empresa ya ha pasado por la transformación, se empieza a consolidar el crecimiento. Siendo este el éxito del conjunto de transformaciones empleadas por la entidad. El crecimiento llega cuando se incrementa la oferta o venta de sus productos y servicios, y estos generan una rentabilidad económica.

3. Competitividad: La competitividad se empieza a consolidar, cuando se han adoptado correctamente estrategias de transformación y estas generan un crecimiento tanto a nivel local, nacional e internacional debido a la satisfacción que se genera en clientes y usuarios. 
Por su parte, Unger manifiesta que la innovación es un ingrediente indispensable en la transformación, crecimiento y competitividad, ya que permite desarrollar y mantener una economía sustentable [4]. Cuando se habla de economía sustentable se hace referencia a la integración de aspectos económicos, sociales y ambientales, con el fin de optimizar la calidad y el bienestar humano. De igual forma Arredondo, Vázquez y de la Garza afirman que la innovación es determinante en la competitividad ya que permite pasar de economías emergentes a economías basadas en el conocimiento y generar un valor compartido y un valor agregado [5]. De acuerdo con lo anterior, si se habla de una empresa que se dedique a la elaboración de libros digitales, no se inventa el libro o el medio digital, sino que permite que este aparato ofrezca un servicio especial para la persona que lo adquiera, que ayude al lector a leer de una manera más didáctica y que le facilite la interpretación de lo que lee, esto genera que el producto o servicio se oferte con calidad y brinde satisfacción al cliente.

\section{Las Tecnologías de la información y comunicación como eje fundamental en la competitividad e innovación empresarial}

Las TIC son fundamentales en el acceso, respaldo y entrega de la información. Hoy en día es común observar como las TIC están incluidas en diferentes mercados como por ejemplo la educación, la salud, el turismo y grandes empresas en la industria nacional e internacional. De igual forma las TIC permiten optimizar diferentes actividades cotidianas, hoy en día las podemos encontrar en aspectos importantes como la radio, la televisión y prensa. Debido a la evolución de las tecnologías han surgido nuevos canales de información como el internet, del cual se han derivado diferentes aplicaciones que actualmente son los más populares, tales como el correo electrónico, las redes sociales y la mensajería instantánea.

Es importante resaltar que las Tecnologías de la Información y Comunicación es uno de los elementos más implementados como fuente de innovación en las empresas ya que permite presentar nuevos productos al mercado u optimizar los que ya están creados. Asimismo, la innovación permite la inclusión de la tecnología como agente de cambio en los diferentes procesos, generando nuevos conocimientos. La utilización de las TIC debe ser un catalizador que permita a las empresas adoptar estrategias que ayuden a mejorar su eficiencia y productividad, y a su vez funcione como un enfoque vinculador que da un valor a los procesos y que permita fomentar la innovación empresarial. Sobre esto, León y Palma afirman que la innovación en las empresas tiene una relación importante con el uso de las TIC, ya que direccionan a las empresas a encontrar oportunidades y ampliar sus ventajas competitivas en el mercado global permitiéndoles impactar en el rendimiento empresarial [6]. De igual forma resaltan que las empresas que deseen tener un mayor impacto de las TIC sobre los procesos de innovación deben adoptar la gestión del conocimiento como pilar fundamental en la implementación de dichas tecnologías.

\section{MetodologíA}

Esta investigación se desarrolló bajo un enfoque cualitativodescriptivo donde se buscó definir los factores que facilitan la competitividad en las empresas y la forma en que la innovación y las TIC influyen en la misma. En primera instancia se utilizaron fuentes bibliográficas con el objetivo de brindar un soporte teórico utilizando los descriptores: innovación, tecnología de la información y comunicación y competitividad.

Finalmente se usó el método de triangulación de datos el cual se define como la obtención de información permitiendo un análisis entre los objetos de investigación [7]. De esta forma se corroboró la información obtenida sobre la innovación y las tecnologías de la información y comunicación como factor de competitividad, los factores claves de éxito de la competitividad, y la competitividad como éxito empresarial. Luego se analizaron unos casos de éxito en donde se identificaron los elementos estudiados. Para el estudio de los casos de éxito fue necesario seguir los lineamientos metodológicos establecidos por Merino [8].

1. Definir los objetivos de la revisión. En esta etapa se identificaron los objetivos de la revisión, donde se estableció abordarlo de forma descriptiva ya que se busca conocer que teorías existen sobre el éxito empresarial utilizando tecnologías de información y comunicación. Un enfoque en los factores de competitividad empresarial también fue analizado en cada caso.

2. Establecer la búsqueda bibliográfica. Este punto definió los criterios bajo los cuales se tomaría en cuenta la inclusión de las fuentes bibliográficas utilizadas en este estudio.

a. Bases de datos y fuentes documentales. La búsqueda de literatura en la elaboración del escrito se basó en diferentes tipos de fuentes bibliográficas, tomándose en cuenta los siguientes tipos de documentos:

1. Fuentes primarias u originales: Es el objetivo de la revisión y proporciona datos de primera mano, son ejemplo de estos: libros, revistas científicas, conferencias, etc.

- Fuentes secundarias: estas ayudan a detectar las referencias necesarias, permiten localizar las fuentes primarias y habitualmente es la estrategia más frecuentemente utilizada. Son compilaciones, resúmenes y listados de referencias publicadas en un área del conocimiento en particular; ejemplo de esto son los catálogos, revisiones sistemáticas, resúmenes, y bases de datos.

- Fuentes terciarias: Cuando no se sabe absolutamente nada del tema se debe recurrir a estas fuentes, que son lugares donde puede obtenerse información para detectar a través de ellas las fuentes primarias o secundarias de interés, ejemplo son las instituciones nacionales $\mathrm{e}$ 
internacionales al servicio de la investigación como bibliotecas, sociedades científicas, etc.

2. Estrategia de la búsqueda. Una vez definida la base de datos, se identificaron las palabras claves con el fin de buscar sinónimos que tuvieran relación con el tema de estudio y de esta forma profundizar la búsqueda.

3. Criterios de selección. Los criterios de selección están determinados por los objetivos de la revisión. En esta etapa se tuvo en cuenta el título, los autores, el resumen y los resultados de cada uno de las revisiones bibliográficas. De igual forma es importante resaltar la lectura crítica con el fin de profundizar en el análisis y enfoque de cada artículo encontrando los casos de éxito para integrar en el escrito.

4. Organización de la información. Se preparó un guion con el fin de orientar el escrito hacia un esquema claro y organizado, esto permitió que la información encontrada tenga un enfoque que lleva a la compresión y posteriormente permita ser usada. En el proceso de estructuración de la información se usó el mapa conceptual y una tabla de análisis y síntesis.

5. Redacción del artículo. Finalmente al tener las ideas claras se procede a plasmarlas, teniendo en cuenta la esencia de un buen escrito según Bobenrieth: claridad, concisión, precisión, sencillez y naturalidad [9]. De manera que sea un proceso lógico que permita expresar los conocimientos encontrados.

En la revisión de artículos y trabajos relacionados con el objeto de estudio, utilizando diferentes fuentes de información, se utilizaron términos en inglés y español con el fin de ampliar la información. Para delimitar la búsqueda se estableció que las publicaciones fueran después de enero del 2014.

\section{TABLA I}

\section{RELACIÓN ENTRE DESCRI'PTORES Y ARTICULOS ENCONTRADOS}

\begin{tabular}{lc}
\hline \hline \multicolumn{1}{c}{ Descriptor } & $\begin{array}{c}\text { Número de investigaciones que } \\
\text { relacionan el descriptor con el éxito } \\
\text { empresarial }\end{array}$ \\
\hline Innovación & 12 \\
$\begin{array}{l}\text { Tecnologías de la información y } \\
\text { comunicación } \\
\text { Competitividad }\end{array}$ & 24 \\
\hline \hline
\end{tabular}

En la tabla I se definen los descriptores utilizados en la búsqueda y cuántos de ellos contribuían al éxito empresarial.

En la consulta realizada a través de Google Scholar, utilizando las palabras y rango de fecha definido, y los descriptores empleados en determinado sector se estableció la relación que estás tenían en el éxito empresarial. Los detalles de la información recolectada se muestran en resumen en la tabla II.
TABLA II

RESUMEN DE LOS ARTÍCULOS ENCONTRADOS

\begin{tabular}{|c|c|}
\hline Ref. & Resumen \\
\hline [10] & $\begin{array}{l}\text { Durante la } \\
\text { investigación } \\
\text { argumenta que la } \\
\text { inversión TIC en las } \\
\text { empresas aumenta la } \\
\text { competitividad y es un } \\
\text { factor de éxito } \\
\text { empresarial. }\end{array}$ \\
\hline [11] & $\begin{array}{l}\text { Realiza un estudio } \\
\text { sobre las necesidades } \\
\text { de los clientes en el } \\
\text { sector turismo y } \\
\text { responder a ellas, a } \\
\text { través del uso de las } \\
\text { TIC convirtiéndolas en } \\
\text { más competitivas }\end{array}$ \\
\hline [12] & $\begin{array}{l}\text { Identifican los factores } \\
\text { que determinan la } \\
\text { competitividad de una } \\
\text { empresa en américa }\end{array}$ \\
\hline
\end{tabular}

[13] Se hace una revisión sobre el concepto de competitividad.

[14] Se analiza la relación entre la competitividad y globalización.

[15] Factores de competitividad en pequeñas empresas.

[16] La innovación como factor de competitividad empresarial en el sector turismo.

[17] Análisis de los procesos de innovación en Colombia

[18] La gestión del conocimiento como un factor de innovación

Se realizó una revisión al concepto de innovación y la influencia de las TIC en la competitividad empresarial.

[20] Se realizó un estudio sobre el impacto de las TIC en la innovación y competitividad empresarial.

[21] La importancia de alinear las TIC con el emprendimiento corporativo para lograr mejorar el impacto de la innovación
Gestión del conocimiento aplicado al Sector empresarial de España

Gestión del conocimiento aplicado al ${ }^{-}$ Sector Turismo de Guatemala

Sector empresarial América Latina

Sector empresarial de Colombia

No aplica (teorías)

Apropiación de las TIC en el sector empresarial de España

Gestión del conocimiento en el sector empresarial de Argentina

Apropiación y gestión del conocimiento de las TIC en el sector empresarial de Colombia

Gestión del conocimiento

No Aplica

Gestión del conocimiento aplicado en el Sector empresarial Europeo

No Aplica (Revisión teórica) 
TABLA II

(CONTINUACIÓN)

\begin{tabular}{|c|c|}
\hline Ref & Resumen \\
\hline$[22]$ & $\begin{array}{l}\text { La importancia de la } \\
\text { gestión del } \\
\text { conocimiento en la } \\
\text { implementación de las } \\
\text { TIC en los procesos } \\
\text { empresariales. }\end{array}$ \\
\hline [23] & $\begin{array}{l}\text { Se realizó un estudio de } \\
\text { como las TIC pueden } \\
\text { optimizar los procesos } \\
\text { empresariales. }\end{array}$ \\
\hline [24] & $\begin{array}{l}\text { Análisis de las TIC en } \\
\text { las empresas de } \\
\text { América Latina. }\end{array}$ \\
\hline [25] & $\begin{array}{l}\text { Se establece una } \\
\text { propuesta respecto a la } \\
\text { estructura de las } \\
\text { organizaciones } \\
\text { empresariales en la } \\
\text { implementación y uso } \\
\text { de las TIC. }\end{array}$ \\
\hline [26] & $\begin{array}{lr}\text { Las tecnologías de la } \\
\text { información } & \text { y } \\
\text { comunicación } & \text { son } \\
\text { facilitador } & \text { de } \\
\text { innovación. } & \end{array}$ \\
\hline
\end{tabular}

[27] Las tecnologías de la información $\mathrm{y}$ comunicación como desarrollo económico empresarial.

[28] Se presentó un modelo en la gestión de las TIC en pequeñas $y$ medianas empresas.

[29] Las tecnologías de la información $\quad \mathrm{y}$ comunicación como fuente creatividad, gestión e innovación.

[30] Se presenta un caso de éxito en Alemania mediante el uso de las TIC en procesos empresariales.

[31] Como afecta las TIC en la competitividad empresarial y en la capacidad de innovación.

[32] Analiza la importancia de la apropiación de las TIC en pequeñas empresas. Caso de estudio: Thika.
Sector empresarial

Valor agregado y gestión de conocimiento de las TIC aplicado en el sector empresarial Colombia

Gestión del conocimiento aplicado al sector empresarial de Colombia

Gestión del conocimiento y aplicación TIC en el Sector empresarial de América y Países Desarrollados

No Aplica (Revisión teórica)

Gestión del conocimiento en el sector empresarial de México

No Aplica (Revisión teórica)

Valor agregado de las TIC aplicado en el sector empresarial de Ecuador

No Aplica (Revisión teórica)

Gestión del conocimiento de las TIC aplicado al Sector empresarial de Alemania

Gestión del conocimiento de las TIC aplicado al Sector empresarial de México

Accesibilidad a las TIC en el Sector empresarial de África
TABLA II

(CONTINUACIÓN)

\begin{tabular}{clc}
\hline \hline Ref & \multicolumn{1}{c}{ Resumen } & Sector empresarial aplicado \\
\hline$[33]$ & $\begin{array}{l}\text { Se realiza un estudio } \\
\text { sobre la eficiencia de } \\
\text { las TIC en su }\end{array}$ & No aplica (Revisión teórica) \\
& aplicación. & \\
& & \\
[34] & Un estudio sobre el & Accesibilidad en las TIC aplicadas al \\
& papel que juegan las & sector empresarial del Salvador \\
& TIC en la \\
& competitividad \\
& empresarial.
\end{tabular}

Sector empresarial de México

[35] Se realiza un estudio sobre la aplicación de las TIC en el sector portuario logrando un cambio en la estructura funcional.

[36] Se realiza un estudio sobre los factores de competitividad empresarial en el sector comercial donde la innovación y la tecnología sobresalen.

[37] Se plantea un caso de éxito en el uso de las TIC como fuente de competitividad empresarial en empresa de calzado.

[38] Se realiza un estudio sobre la efectividad de las TIC en procesos administrativos en Pymes.

[39] Se realiza un estudio sobre la importancia de la gestión de las TIC para la competitividad empresarial.

[40] Implementación y uso de las TIC según el tipo de empresa para su aprovechamiento.

[41] Se realizó un análisis empresarial sobre la influencia de las TIC en empresas dedicadas al sector de servicios.

[42] Se presentó un estudio sobre el uso que le dan las PYMES de Calzado a las tecnologías de la información comunicación
Accesibilidad en las TIC aplicados al sector empresarial de México

Gestión del conocimiento en el Sector empresarial de Colombia

Apropiación de las TIC en el Sector empresarial de Colombia

Gestión del conocimiento de las TIC en el sector empresarial de México

Gestión del conocimiento de las TIC en el Sector empresarial de Colombia

Apropiación y Gestión del conocimiento de Las TIC en el Sector empresarial de Perú 
Durante la revisión bibliográfica realizada se logró evidenciar como las empresas de diferentes sectores y magnitud pueden llevar procesos de innovación internos y externos por medio de las tecnologías de la información y comunicación permitiéndoles mejorar su productividad y consolidándolas en el mercado global. ¿Cómo las empresas que incorporan estrategias de innovación por medio de las tecnologías de la información y comunicación logran tener éxito en ser competitivas?

En los documentos estudiados las tecnologías de la información y comunicación y la innovación, han sido un factor de éxito en diferentes sectores productivos, la figura número 2, muestra los factores que resultaron determinantes y que demuestran que influyen en el éxito en la implementación de la innovación y las tecnologías de la información y comunicación en los procesos empresariales incidiendo en su competitividad.

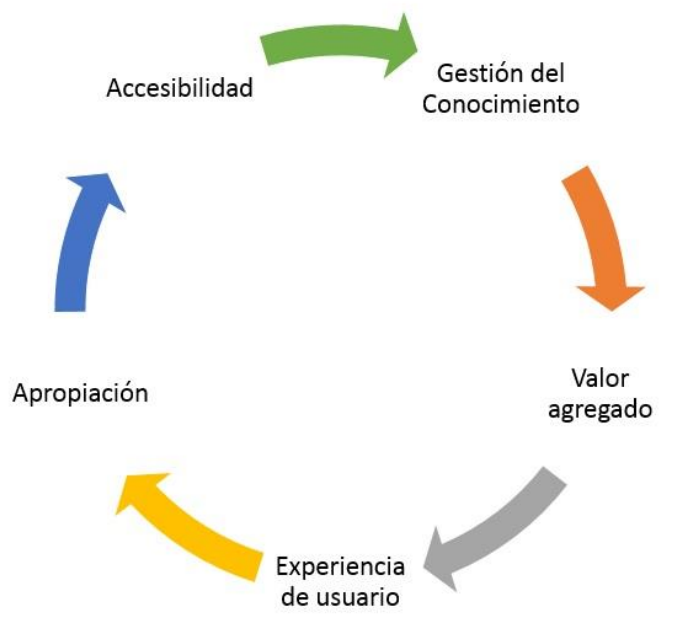

Fig. 2. Factores clave de éxito empresarial en la incorporación de la innovación y las Tecnologías de la información y comunicación

Los casos que se describen a continuación permiten identificar dichos factores clave en diferentes sectores empresariales que hoy en día no paran de generar nuevo conocimiento con el fin de seguir consolidándose en el mercado nacional e internacional como las empresas con alta competitividad en el mundo. Competitividad empresarial lograda e incrementada con la adopción de políticas de innovación y la apropiación de las tecnologías de la información y comunicación.

A. La gestión del conocimiento y las tecnologías de la información y comunicación como fuente de competitividad empresarial. Caso Fundación Cardiovascular Colombia

En Colombia la fundación cardiovascular (FCV) durante los últimos años se ha consolidado como uno de los complejos más importantes del país. Se encuentra en la categoría nivel cuatro de complejidad y es la primera institución en el país acreditada por la Joint Commission International, sello que avala altos estándares de calidad certificada. El Hospital Internacional de Colombia (HIC) hace parte de la fundación cardiovascular de
Colombia (FVC). El Ránking de Clínicas y Hospitales publicado por el sitio web Clúster Salud - América Economía, realizado en el año 2017 en donde 49 hospitales participaron, el Hospital Internacional de Colombia (HIC) ubicado en la ciudad de Bucaramanga ocupó el octavo lugar en américa como una de las entidades que tienen la capacidad de generar, obtener y difundir los saberes médicos [43]. ¿Pero qué aspectos influyeron en la consolidación del Hospital Internacional de Colombia como uno de los mejores de América? Uno de los aspectos que influyeron en la internacionalización del HIC fue la adopción de la competitividad como gestión empresarial. El HIC adoptó estrategias de innovación en conjunto con las TIC y crearon una herramienta denominada Sistema Hospitalario Integrado para Pacientes (SHIPP), la plataforma ofrece una serie de aplicaciones como el ingreso a redes sociales, correo electrónico, acceso a música, películas y a telefonía internacional [44]. De igual forma esta aplicación permite el seguimiento del paciente por parte del personal médico, y finalmente serán implementadas funcionalidades de domótica que le permitirá al paciente controlar la iluminación de la habitación, el clima y los llamados de enfermería. Se afirma que el Hospital Internacional de Colombia (HIC) es uno de los establecimientos colombianos que le ha apostado a la adopción de las TIC, permitiéndoles continuar consolidándose como una entidad competitividad a través de la innovación mejorando su capacidad de servicio específicamente en el sector salud [45].

Por otra parte, las TIC han jugado un papel importante en la innovación de los procesos y productos claves del sector turístico, convirtiéndose en un canal de comunicación que busca establecer una relación con los clientes y de igual forma busca facilitar la interconexión a nivel mundial. Hoy en día no sólo facilita la interconexión, sino que mejora la experiencia turística en el mundo.

\section{B. La experiencia de usuario en el turismo y la importancia en el éxito digital. Caso Airbnb}

Airbnb es una aplicación que busca promover el turismo en las ciudades del mundo, a través de una red digital. Esta herramienta permite que las personas publiquen y realicen reservas de alojamiento por internet. Este sistema ha sido uno de los más exitosos del mundo, ya que permite que se realicen intercambios de bienes y servicios entre particulares por medio de plataformas digitales. La empresa ha pasado de ganar 200 euros semanales a convertirse en una de las plataformas que ha revolucionado el turismo [46]. El modelo de Airbnb tiene un potencial competitivo en el ámbito de la economía colaborativa en el sector turístico [47]. La aplicación Airbnb ofrece a los usuarios no solo la posibilidad de encontrar cualquier alojamiento, sino que permite acercarse a la cultura de la ciudad a la que visita, ya que el usuario puede convivir en el hogar con personas nativas de la región, así como también puede alquilar, casas de árbol, iglús, geo domos, molinos entre otras cosas mejorando la experiencia de usuario de las personas que hacen turismo. De igual forma, las TIC han simplificado los procesos y optimizado los costes tanto para el consumidor como para la compañía. El éxito que ha tenido esta aplicación está estrechamente relacionado con la tecnología, ya que ofrece a los 
usuarios nuevas experiencias en el acceso de servicios y productos permitiéndoles a las empresas mejorar la competitividad por medio de la innovación y adopción de las TIC.

C. La accesibilidad en las tecnologías de la información y comunicación un factor de inclusión y desarrollo. Caso sector agricultura, Holanda

La agricultura es uno de los sectores primarios más importantes para el ser humano ya que les permite satisfacer sus necesidades por medio de la producción de alimentos. En la producción agrícola intervienen factores como: el clima, el suelo, y la inversión económica. De igual forma, la agricultura tiene un alto potencial en el crecimiento sostenible y sobre todo en la economía. Holanda es uno de los países que ha tenido éxito en las exportaciones de productos agroalimentarios, esto significa que el sector agrícola está multiplicando la productividad, gracias a las nuevas técnicas de desarrollo e innovación. Según Nieves [48] Holanda ha revolucionado el sector de la agricultura, ha exportado unos 80.000 millones de euros permitiéndole aportar al crecimiento económico del país. ¿Pero cómo han logrado este crecimiento? Las políticas agrarias del gobierno de los países bajos giran en torno a la innovación tecnológica, permitiendo que los agricultores reciban subvenciones para que modernicen sus cultivos. La gestión del conocimiento juega un papel importante en la adopción de las TIC en la agricultura holandesa por esta razón crearon una entidad encargada de brindar consejos a los campesinos sobre que herramientas y estrategias TIC implementar en sus cultivos, esta entidad tiene por nombre oficina de concejo agrícola. La innovación, la gestión del conocimiento y las TIC como política de estado en el sector de la agricultura ayuda a mejorar la productividad y la competitividad. Para Nowak y Kaminska [49] la competitividad en la agricultura holandesa se encuentra relacionada con la productividad, la innovación y las TIC por esta razón es uno de los países europeos más competitivos.

\section{Transformar el sector transporte. La experiencia de usuario más innovadora y exitosa. Caso Uber}

Uno de los sectores al que también le ha apostado a la tecnología es el sector de transporte. Debido a la globalización se hace necesario que medios de transporte permitan a las personas trasladarse, a sus hogares, trabajos o sitios de estudios. Por esta razón, nace el transporte público, el cual es un sistema integral de medios de transporte de uso generalizado. La plataforma Uber surge gracias a la innovación y el uso de las TIC, es un medio que facilita la comunicación entre un conductor y un usuario que desea un conductor privado. Esta aplicación esta cambiado la manera en que las personas se pueden desplazar por las ciudades, ya que permite de manera simple, rápida y confiable conectar a conductores con usuarios que requieran de un servicio de traslado a través de la tecnología. El usuario vive una serie de experiencias innovadoras a través del uso de la Aplicación Uber, para Muñoz [50] la fórmula para alcanzar la competitividad es el uso de las
TIC, y menciona el caso de Uber y otras empresas que han optado por el alto uso de contenido tecnológico en sus procesos empresariales. Uber no para de trabajar en la competitividad, y ha proyectado para el 2025 ofrecer un servicio de transporte de viajeros por el aire por medio de vehículos VTOL, estos tienes semejanzas a los aviones, pero los diferencian de ellos la forma de despegar y aterrizar ya que los realizan de manera vertical [51].

\section{E. La apropiación de las Tecnologías de la Información y Comunicación como factor de innovación en la educación han tocado las puertas para quedarse. Caso Sector Educación, Singapur}

Cuando hablamos de educación, nos referimos a los procesos que adquiere una persona. Estos procesos pueden ser culturales y conductuales. Singapur es la capital de la República de Singapur, nació siendo una pequeña isla con el paso de los años hasta la actualidad se ha transformado en un país ejemplo de modelo global de innovación y éxito económico. Hoy la ciudad de Singapur es la más productiva y competitiva de Asia y del mundo. Este país maneja el mejor sistema de educación según el programa para la Evaluación Internacional de Alumnos (PISA), pero ¿cuál ha sido el éxito? Por supuesto uno de los factores clave es la innovación, este país está acostumbrado a implementar la innovación en cada uno de sus procesos, posiciónalo por encima de muchos países del mundo. Para Falco [52] las TIC facilitan que las organizaciones se adapten al cambio que genera la globalización, y de esta manera en la educación los estudiantes prefieren los modelos de enseñanza que empleen tecnología ya que fomentan el aprendizaje a través de la experiencia, por esta razón Singapur se ha convertido en pionero en la adopción de las TIC como método de enseñanza e innovación. El método de enseñanza de Singapur se ha enfocado no solo en el uso de las TIC como un factor de innovación en sus aulas y por supuesto en el sistema educativo, sino que también ha culturizado a los niños de tres años con las Tecnologías, enseñándoles a programar videojuegos, circuitos y drones. Esto significa que no solo son un país que consume tecnología, sino que también crea su propia tecnología. Las TIC han jugado un papel fundamental desde principio a fin en la educación de Singapur, además, en la metodología de enseñanza, este país asiático usa métodos que involucran a las Tecnologías de la Información y Comunicación lo cual permite que los estudiantes aprendan de manera didáctica, ya que el alumno permite plantear sus propias formas de pensar y de desarrollar la lógica, y el profesor solo se encarga de observar como realizan estos procesos de aprendizaje. Es necesario resaltar que las TIC en el uso educativo es una política de estado prioritaria, ya que contribuyen al desarrollo, la innovación y la competitividad del sistema educativo.

Las experiencias descritas anteriormente permiten reflexionar ante los factores de competitividad reinantes en la actualidad en donde se puede reconocer que la gestión del conocimiento le permitió al Hospital Internacional de Colombia gestionar los recursos humanos como su mayor activo, y sustenta su competitividad a través de la capacidad de compartir, difundir y explotar la información de sus pacientes, logrando 
satisfacción, participación, compromiso, motivación por parte de los empleados y pacientes convirtiéndose en una entidad competitiva. Airbnb usó el valor agregado como fuente de innovación, ya que no solo desarrollaron una aplicación de alquiler vacacional, por su parte agregaron características extra que ofrecen un valor comercial, la cual los convierte en una empresa competitiva en uno de los sectores con más demanda en el mundo, incentivando el emprendimiento para las personas que se animan a ofrecer sus servicios como un intercambio de experiencias desde la comodidad de su hogar. La experiencia de usuario es importante en cualquier producto o servicio que ofrece una empresa, ya que permite establecer una relación entre el cliente y la empresa de manera emocional, Uber a través de su plataforma digital logró capturar sus clientes y convertirse en una de las empresas más competitivas e innovadoras por medio de este factor, ya que lograron no sólo solucionar un problema de transporte sino asegurar la satisfacción, confort y seguridad, entre las personas que se movilicen por las ciudades. En los procesos de aprendizaje Singapur ha optado por apropiar a los niños y niñas en el uso de las Tecnologías de la información y comunicación, logrando mejorar la educación y convertirse en el país que mejor maneja el sistema de educación. La accesibilidad de las Tecnologías de la información y comunicación, permitieron que en Holanda los campesinos mejoraran su productividad, y se posicionaran como el país más competitivo en la agricultura. Es importante mencionar que, en este último caso, se estableció una relación entre la gestión del conocimiento, la accesibilidad y la apropiación ya que los campesinos a través del estado logran adquirir los conocimientos sobre la tecnología para finalmente utilizarla en sus cultivos logrando mejorar la competitividad.

\section{CONCLUSIONES}

Las TIC y la innovación es la forma de hacer más productivas las empresas, y llevarlas al campo de la competitividad a nivel internacional. La libertad empresarial es un nuevo concepto que se debe empezar a desarrollar en las empresas ya que para ellas no deben existir límites, ni fronteras que permitan intercambios mundiales. Las Tecnologías de la Información y Comunicación permiten que las empresas evolucionen y logren una competitividad sostenible que permite el desarrollo, hoy se habla de TIC, innovación y competitividad como la clave del éxito empresarial sin importar el tipo de industria o el tamaño, debido a que estás son herramientas transversales que permiten agregar valor a las empresas e instituciones de diferentes sectores económicos.

Asimismo, este artículo permitió identificar los siguientes factores como influyentes en el éxito de la implementación de la innovación y las tecnologías de la información y comunicación haciendo que estén alineadas a los procesos y objetivos empresariales.

- Gestión del Conocimiento. La gestión del conocimiento busca potencializar los procesos innovadores en las empresas con el fin de convertirse

en estrategia efectiva para alcanzar la competitividad empresarial.

- Valor agregado. El valor agregado es una característica de los productos o servicios ofrecidos por las empresas que busca generar valor en la percepción del consumidor. Este factor puede ser determinante en el éxito, ya que se encarga de posicionar la empresa frente a la competencia.

- Experiencia de usuario. La experiencia de usuario es el proceso que lleva a cabo el usuario cuando adquiere un producto o servicio, los aspectos emocionales juegan un papel importante ya que influye en la relación que se puede establecer entre el producto y el usuario.

- Apropiación. Cuando se implementan estrategias mediante las tecnologías de la información y comunicación es importante que todas las personas que intervengan desarrollen habilidades $y$ competencias tecnológicas que les permita apropiarse de los beneficios que estas herramientas ofrecen al interior de la empresa con el fin de incidir en el desarrollo de procesos innovadores.

- Accesibilidad. La accesibilidad permite garantizar que cualquier persona a través de cualquier medio pueda acceder a las tecnologías de la información y comunicación, por esta razón es importante que las empresas a la hora de desarrollar un producto o servicio incluyan el mayor número de personas independientemente de las limitaciones personales o limitaciones de su entorno y de esta forma satisfacer sus necesidades.

Finalmente, las empresas deben conocer la capacidad que manejan al interior de la empresa para proporcionar alguna ventaja con respecto a los procesos de innovación, del mismo modo es necesario que las empresas conozcan que potencial tienen para exigirse a sí mismas y de esta manera competir en el mercado.

\section{REFERENCIAS}

[1]

J. Garcia Guiliany, S. Duran, E. Cardeño Pórtela, R. Prieto Pulido, E. García Cali, and A. Paz Marcano, "Proceso de planificación estratégica: Etapas ejecutadas en pequeñas y medianas empresas para optimizar la competitividad," Rev. Espac., vol. 38, no. November, pp. 16-30, 2017.

M. Ibarra, L. González, and M. de R. Demuner, "Competitividad empresarial de las pequeñas y medianas empresas manufactureras de Baja California," Estud. Front., vol. 18, no. 35, pp. 107-130, 2017. DOI: 10.21670/ref.2017. 35.a06
G. C. López-Torres, G. Maldonado Guzmán, S. Y. Castro Pinzón, and R. García Ramírez, "Colaboración y actividades de innovación en Pymes," Contaduria y Adm., vol. 61, no. 3, pp. 568-581, 2016. DOI: 10.1016/j.cya.2015.05.016 
[4] K. Unger, "Innovación, competitividad y rentabilidad en los sectores de la economía mexicana. /Innovation, Competitiveness and Profitability in the Mexican Economy," Gestión y Política Pública, vol. 27, no. 1, pp. 3-37, 2018.

F. Arredondo Trapero, J. C. Vázquez Parra, and J. de la Garza, "Factores de innovación para la competitividad en la Alianza del Pacífico. Una aproximación desde el Foro Económico Mundial," Estud. Gerenciales, vol. 32, no. 141, pp. 299-308, 2016. DOI: 10.1016/j.estger.2016.06.003

O. León and E. Palma, "Aplicación de las Tecnologías de Información y comunicación en los procesos de innovación empresarial. Revisión de la literatura," I+D Rev. Investig., vol. 11, no. 1 , pp. 156-166, 2018.

T. Alzás García, L. M. Casa García, R. Luengo González, J. L. Torres Carvalho, and S. V. Catarreira, "Revisión metodológica de la triangulación como estrategia de investigación," Investig. Cual. en Ciencias Soc., vol. 3, pp. 639-648, 2016.

[8] A. Merino Trujillo, "Como escribir documentos científicos (Parte 3). Artículo de revisión," Salud en Tabasco, vol. 17, pp. 36-40, 2011.

M. A. Bobenrieth Astete, El Artículo científico original: estructura, estilo y lectura crítica. Escuela Andaluza de Salud Pública, 1994.

C. Piñeiro Sánchez, P. De Llano Monelos, and M. Rodríguez López, "Las TIC como inductores de competitividad y facilitadores del éxito empresarial,” Ijisebc, vol. 3, no. 1, pp. 8-26, 2016.

C. Rodríguez Monrroy and S. Rivera Ramos, "Utilización de las TIC y valoración de la competitividad de las empresas turísticas en Guatemala," Rev. Tur. Econ. y Negocios, vol. 2, no. 21, pp. 116-132, 2016.

D. Molina Ycaza and A. M. Sánchez-Riofrío, "Factores de competitividad orientados a la pequeña y mediana empresa (PYME) en Latinoamérica: revisión de la literatura," Rev. San Gregor., no. 15, pp. 104-111, 2016.

[13] M. A. Cabezas Arboledas and G. E. Reyes Ortiz, "Factores críticos para la competitividad colombiana: desde la cara de la competitividad sistémica," Universidad del Rosario, Administración de Negocios Internacionales, 2017.

[14] J. G. Vargas Hernández, "Las reglas cambiantes de la Competitividad Global en el Nuevo Milenio. Las competencias en el Nuevo Paradigma de la Globalización,” Rev. Iberoam. Educ., vol. 1, pp. 121, 2011.

[15] Á. Díaz-Chao, J. Sainz-González, and J. Torrent-Sellens, "The competitiveness of small network-firm: A practical tool," J. Bus. Res., vol. 69, no. 5, pp. 1769-1774, 2016. DOI: 10.1016/j.jbusres.2015.10.053

[16] J. D. Martins Aires and C. Amorim Varum, "La investigación sobre la medición de la innovación en las empresas de turismo," Estud. y Perspect. en Tur., vol. 27, no. 0327-5841, pp. 102-120, 2018.

[17] J. Robledo Velásquez and F. Malaver Rodríguez, "Los datos, la información y el conocimiento sobre la innovación en Colombia y sus perspectivas de desarrollo," in Encuestas, datos y descubrimiento de conocimiento sobre la innovación en Colombia, 2016, p. 366.

[18] L. H. Peña Vargas, C. A. Vega Durán, and J. G. Castellanos Méndez, "Innovación y Gestión del Conocimiento para el Incremento de la Productividad Empresarial.," Memorias, vol. 14, no. 26, pp. 1-41, 2016. DOI: $10.16925 / \mathrm{me} . \mathrm{v} 14 \mathrm{i} 26.1571$
[19] A. Marín García and I. Gil Saura, "Innovar en el comercio minorista: Influencia de las TIC y sus efectos en la satisfacción del cliente," Cuad. Gest., vol. 17, no. 2, pp. 109-134, 2017. DOI: 10.5295/cdg.150556am

[20] S. Gërguri-Rashiti, V. Ramadani, H. Abazi-Alili, L.-P. Dana, and V. Ratten, "ICT, Innovation and Firm Performance: The Transition Economies Context," Thunderbird Int. Bus. Rev., vol. 59, no. 1, pp. 93-102, Jan. 2017. DOI: 10.1002/tie.21772

M. Yunis, A. Tarhini, and A. Kassar, "The role of ICT and innovation in enhancing organizational performance: The catalysing effect of corporate entrepreneurship," J. Bus. Res., vol. 88, no. June 2017, pp. 344-356, Jul. 2018. DOI: 10.1016/j.jbusres.2017.12.030

D. Oliveros Contreras and G. M. Martínez, "Efecto de las TIC sobre la gestión de las empresas hoteleras afiliadas a Cotelco de Bucaramanga (Santander, Colombia)," Rev. EAN, no. 83, pp. 15-30, 2017. DOI: $10.21158 / 01208160 . n 83.2017 .1827$

A. Aguilera-Castro, G. P. Ávila-Fajardo, and O. J. Solano-Rodríguez, "Las TIC en la formulación estratégica de las pymes de Santiago de Cali - Colombia," Entramado, vol. 13, no. 1, pp. 102-111, 2017. DOI: $10.18041 /$ entramado.2017v13n1.25106

D. J. Quiroga-Parra, J. Torrent-Sellens, and C. P. Murcia-Zorrilla, "Las tecnologías de la información en América Latina, su incidencia en la productividad: Un análisis comparado con países desarrollados," DYNA, vol. 84, no. 200, pp. 281-290, Jan. 2017. DOI: $10.15446 /$ dyna.v84n200.60632

G. E. Cano-Pita, "Las TICs en las empresas evolución de la tecnología y cambio estructural en las organizaciones," Dominio las Ciencias, vol. 4, pp. 206-217, 2018.

H. Cuevas-Vargas, S. Estrada, and E. Larios-Gómez, "The Effects of ICTs As Innovation Facilitators for a Greater Business Performance. Evidence from Mexico," Procedia Comput. Sci., vol. 91, no. Itqm, pp. 47-56, 2016. DOI: 10.1016/j.procs.2016.07.040

S. Mian, W. Lamine, and A. Fayolle, "Technology Business Incubation: An overview of the state of knowledge," Technovation, $\begin{array}{llll}\text { vol. } 50-51, \quad \text { pp. } 1-12, & 2016 . & \text { DOI: }\end{array}$ 10.1016/j.technovation.2016.02.005

[28] M. Sluzarczyk Antosz, "Modelo de Gestión de las Tic para las Pymes de Riobamba (Ecuador)," 3C TIC Cuad. Desarro. Apl. a las TIC, vol. 6, no. 4, pp. 1-18, Jan. 2018. DOI: 10.17993/3ctic.2017.58.1-18

Y. A. Borja López, G. F. Gutiérrez Constante, and L. G. Cevallos Black, "Efectos Del Consumo Digital En La Educación Latinoamericana," Rev. Publicando, vol. 4, no. 11(1), pp. 690-703, 2015.

C. Schröder, "New Insights on the ICT Sector in GermanyEmpirical Studies on ICT Firm Growth and Knowledge Spillover, ICT Cooperation Networks, and Early Stage Venture Capital Investments," Universität Wuppertal, Fakultät für Wirtschaftswissenschaft/Schumpeter School of Business and Economics, 2018.

[31] L. E. Valdez Juarez, R. Limon Ulloa, and E. A. Ramos Escobar, "Las tic, la innovación y los efectos en la competitividad de la pyme," Rev. Int. Adm. y Finanz., vol. 9, no. 7, pp. 87-108, 2016.

S. O. Mokaya and E. W. Njuguna, "Adoption and Use of Information and Communication Technology (Ict) By Small Enterprises in Thika Town, Kenya,” Sci. Conf. Proc., pp. 498-504, 2017.
N. Roztocki and H. R. Weistroffer, "Conceptualizing and 
Researching the Adoption of ICT and the Impact on Socioeconomic Development," Inf. Technol. Dev., vol. 22, no. 4, pp. 541-549, Oct. 2016. DOI: $10.1080 / 02681102.2016 .1196097$

C. M. R. Argueta, "La tecnología como factor de competitividadrecientes indicadores internacionales de desempeño," Entorno, no. 61, pp. 68-75, 2016. doi.org/10.5377/entorno. v0i61.6131

[35] J. A. Cortes Méndez, J. A. Páez Páez, and J. O. Lozano, “Aplicacion de las TIC en los sistemas de gestion de las Pyme del sector portuario," Rev. Electron. Redes Ing., vol. 7, no. 1, p. 18, 2016. DOI: 10.14483/udistrital.jour.redes.2016. 1.a05

[36] R. I. Ramírez Molina and D. E. Ampudia Sjogreen, "Factores de Competitividad Empresarial en el Sector Comercial," Rev. Electrónica Cienc. y Tecnol. del Inst. Univ. Tecnol. Maracaibo, vol. 4, no. October, 2018.

[37] L. Manzo Martínez and G. G. Alfaro Calderón, "El tic's como fuente de ventaja competitiva en las empresas exportadoras de la industria del calzado," Red Int. Investig. en Compet., vol. 9, pp. 438-457, 2016.

[38] S. Riascos Erazo, A. Aguilera Castro, and O. Solano Rodriguez, "Efectividad de las TIC en los Procesos Administrativos de las PYMES de Santiado de Cali - Colombia," Rev. Gerenc. Tecnológica Informatica., vol. 14, no. 40, pp. 17-30, 2016.

[39] J. F. Castellanos Galeano, M. H. Loaiza, and C. A. Cuesta Iglesias, "Importancia de las TIC para la competitividad de las Pymes en Colombia," Puente, vol. 10, no. 1, pp. 93-99, 2016. DOI: 10.18566/puente.v10n1.a10

[40] M. A. Ibarra Cisneros, L. A. González Torres, and K. E. Cervantes Collado, "El aprovechamiento de las TIC en empresas pequeñas y medianas de Baja California, México: el caso del sector manufacturero,” Rev. Int. Econ. y Gestión las Organ., vol. 3, pp. 4557, 2016.

[41] H. A. Botello Peñaloza, A. C. Pedraza Avella, and O. E. Contreras Pacheco, "Análisis empresarial de la influencia de las TIC en el desempeño de las empresas de servicios en Colombia," Rev. Virtual Univ. Católica del Norte, vol. 2, no. 6, pp. 3-15, Sep. 2015. DOI: 10.1111/aos. 13438

[42] J. Cholán León and E. Cano Urbina, "Tecnologías de Información y Comunicación y su Incidencia en la Competitividad de las Mypes de Calzado del distrito El Porvenir," Cienc. y Tecnol., vol. 12, no. 3, pp. 255-271, 2017.

"Ránking de Clínicas y Hospitales: Estos son los mejores de América Latina," 23/11/2017. [Online]. Available:https://clustersalud.americaeconomia.com/gestionhospitalaria/ranking-de-clinicas-y-hospitales-estos-son-los-mejoresde-america-latina. [Accessed: 22-Nov-2018].

[44] E. C. Rodríguez, A. Mojica Cueto, and A. Charris Fontanilla, "Clúster de turismo de salud en Colombia: referentes para la competitividad," Duazary Rev. Int. Ciencias la Salud, vol. 15, pp. 307-323, 2018. DOI: 10.21676/2389783X.2423

[45] S. Angulo, "Colombia avanza en Tecnología para la salud," 27/10/2016, 2016. [Online]. Available: http://www.enter.co/culturadigital/salud-digital/colombia-avanza-en-tecnologia-para-la-salud/. [Accessed: 22-Nov-2018].

[46] BBVA, “Airbnb, un caso de éxito de Design Thinking," 14 de octubre del 2015, 2015. [Online]. Available: https://www.bbva.com/es/podcast-una-banca-mejor-para-unasociedad-mejor/. [Accessed: 22-Nov-2018].
R. J. Díaz Armas, D. Gutiérrez Taño, and F. J. García Rodríguez, "AIRBNB Como nuevo modelo de negocio disruptivo en la empresa turística: Un análisis De su potencial competitivo a partir de las opiniones de los usuarios,” Análisis Turístico, vol. 12, 2014.

[48] V. Nieves, "Holanda revoluciona la agricultura: exporta más alimentos que España y Francia juntas con métodos sostenibles," $2018 . \quad$ [Online]. Available: https://www.eleconomista.es/economia/noticias/8885958/01/18/Hol anda-revoluciona-la-agricultura-exporta-mas-alimentos-que-Espanae-Francia-juntas-con-metodos-sostenibles.html. [Accessed: 22-Nov2018].

[49] A. Nowak and A. Kaminska, "Agricultural competitiveness: The case of the European Union countries," Agric. Econ. (Zemědělská Ekon., vol. 62, no. No. 11, pp. 507-516, 2016. DOI: 10.17221/133/2015AGRICECON

[50] D. N. Muñoz Bastardo, "Organización de Empresas: Nuevo Contexto Empresarial y Competitivo," Universidad de Valladolid, Facultad de Ciencias Económicas y Empresariales, 2017.

[51] A. M. Luzardo, "Uber quiere volar con un nuevo servicio: Uber Elevate.," 06-02-2017, 2017. [Online]. Available: http://www.enter.co/chips-bits/apps-software/uber-quiere-volar-conun-nuevo-servicio-uber-elevate/. [Accessed: 05-Dec-2018].

[52] M. Falco, "Reconsiderando las prácticas educativas: TICs en el proceso de enseñanza-aprendizaje," Tendencias Pedagógicas, vol. 29, pp. 59-76, 2017. DOI: 10.15366/tp2017.29.002

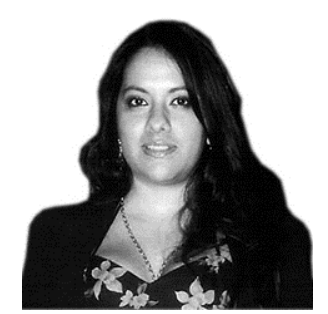

Mary Carlota Bernal Jiménez, Ingeniero en informática, Universidad Nacional Experimental del Táchira, San Cristóbal, Estado Táchira, Venezuela. Magister en Ciencias de la Computación, Universidad de los Andes, Estado Mérida, Venezuela. Doctorando en Ingeniería Informática y matemáticas para la seguridad Universidad Rovira i Virgili, Tarragona, España. Personal académico de la Universidad Simón Bolívar, Cúcuta, Colombia. Personal académico en pregrado y postgrado de la Universidad Nacional Experimental del Táchira, San Cristóbal, Venezuela. Investigador activo IngeBioCaribe Universidad Simón Bolívar. Investigador activo Laboratorio de Investigación y Desarrollo en Informática Universidad Nacional Experimental del Táchira. Áreas de investigación: inteligencia artificial, inteligencia de negocios, innovación, gestión de proyectos de tecnología, arquitecturas de información y empresariales, ciudades y salud inteligente.

ORCID: http://orcid.org/0000-0001-7565-2909 


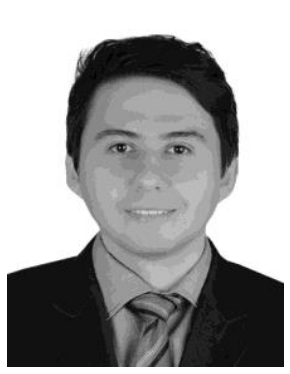

Diego Libardo Rodríguez Ibarra, Ingeniero de sistemas actualmente hago parte del Capital Humano de Alto Nivel para el Departamento de Norte de Santander como joven investigador de Colciencias. Pertenezco al grupo de ingestigacion IngeBioCaribe con linea de invesigacion en Gestion de la Tecnología informatica. $\mathrm{He}$ desarrollado proyectos de investigación en la Universidad Simón Bolívar obteniendo reconocimientos a nivel Nacional. De igual forma, he logrado consultorias en empresas de Cúcuta logrando avances significativos en en la gestion de las tecnologias de la informacion y la comunicación. ORCID: http://orcid.org/0000-0002-7668-2701 